\title{
La interpretación primitiva del diseño en la fábrica argentina de helicópteros Cicaré S.A. (1956-2015)
}

| Fecha de recibido: 08 de agosto del 2020 | Fecha de aprobación: 25 de septiembre del 2020 |

\author{
Leandro Colombano \\ Magíster en Ciencia, Tecnología e Innovación \\ Universidad Nacional de Río Negro, Argentina \\ Rol de investigador: teórico, experimental y escritura \\ https://orcid.org/0000-0002-1574-3347 \\ $\bowtie$ leo-svr@hotmail.com
}

\section{Diego Sebastián Aguiar \\ Doctor en Ciencias Sociales}

Universidad Nacional de Río Negro, Argentina Rol de investigador: teórico, experimental y escritura https://orcid.org/0000-0002-5569-8384 $\bowtie$ daguiar@unrn.edu.ar 


\section{La interpretación primitiva del diseño en la fábrica argentina de helicópteros Cicaré S.A. (1956-2015)}

Resumen: el conocimiento tecnológico generado en una empresa puede definirse como el conjunto de conocimientos provenientes del diseño, análisis, construcción y ensayos de sus productos tecnológicos, innovaciones y procesos. Las características de este tipo de conocimiento hacen que su conservación y transmisión sea compleja, particularmente, debido a su alto componente tácito. Por ello es importante indagar sobre sus características para facilitar su creación, gestión, conservación y transmisión. El objetivo de este estudio es identificar la metodología de trabajo en las actividades asociadas al diseño y fabricación de helicópteros. De igual manera, se abordará la gestión del conocimiento generado y su evolución en el tiempo en una empresa tomada como caso de estudio. Las prácticas y roles que desempeñan cada uno de los actores de la empresa son aspectos significativos en el análisis del caso. Se ha recurrido al análisis técnico de todos los helicópteros desarrollados por la empresa Cicaré S.A., que, como particularidad, no ha contado con registros de actividades o estados patrimoniales en el tiempo. Las entrevistas con personas relacionadas con la firma actual y su historia han sido fundamentales para cumplir con el objetivo. Cicaré S.A. es una empresa argentina de aeronáutica, reconocida internacionalmente, dedicada al desarrollo y fabricación de helicópteros. Es considerada un referente en la industria por varias innovaciones, empleadas hoy en todo el mundo, que ha desarrollado para este tipo de aeronaves. El primer vuelo vertical en Sudamérica, el desarrollo de uno de los primeros helicópteros ultralivianos y un simulador de vuelo real controlado son algunas innovaciones que han destacado a la firma. El presente estudio identifica que en la empresa coexisten dos metodologías de diseño e innovación aplicadas a la tecnología aeronáutica. Por un lado, cuenta con un departamento de producción que mediante metodología ingenieril estándar produce helicópteros en serie. Por otro lado, cuenta con un departamento de investigación y desarrollo que aplica metodología de análisis de prototipos como medio de generación de innovaciones y mejora de sus productos.

Palabras clave: aeronáutica; conocimiento; diseño; helicóptero; ingeniería; prototipo.

Abstract: The technological knowledge generated by a company can be defined as the set of information coming from the design, analysis, construction and testing of its technological products, innovations, and processes (McGinn, 1991). This type of knowledge entails characteristics that make it difficult to be preserved and transmitted, particularly due to its high tacit component (Polanyi, 1966). For this reason, it is important to investigate the characteristics of this class of knowledge in order to facilitate its creation, management, conservation, and transmission. Therefore, the objective of this study is to identify the work methodology of activities associated with the design and manufacturing of helicopters, as well as the knowledge management generated and its evolution path over time in a company selected as case study. The practices and roles played by each of the actors within the company are also significant aspects to be considered. The technical analysis of all the helicopters developed by the company Cicaré S.A. has been used as source of information; although, in particular, there are not available records of this company's activities or financial statements over time. Interviews with people related to the current firm and its history have been fundamental. Cicaré S.A. is an Argentinian aeronautical company working in the development and manufacturing of internationally recognized helicopters. It is considered a benchmark in the industry for the innovations it has developed on this type of aircraft, which are used today throughout the world. The first vertical flight in South America, the development of one of the first ultralight helicopters and a real controlled flight simulator are some of the firm's main innovations. This study seeks to identify the coexistence of two design and innovation methodologies applied to aeronautical technology by this company. On the one hand, there is a production department that, using standard engineering methodology (Mott, 1995), produces serial helicopters. Besides, a research and development department applies prototype analysis methodology (Gero, 1990) looking for innovations and product improvements.

Keywords: Aeronautics, knowledge, design, helicopter, engineering, prototype. 


\section{A interpretação primitiva do desenho na fábrica argentina de helicópteros Cicaré S.A. (1956-2015)}

Resumo: O conhecimento tecnológico gerado em uma empresa pode ser definido como o conjunto de conhecimentos a partir do projeto, análise, construção e teste de seus produtos tecnológicos, suas inovações e seus processos (McGinn, 1991). Esse tipo de conhecimento possui características que dificultam sua preservação e transmissão, principalmente devido ao seu alto componente tácito (Polanyi, 1966). Por isso, é importante pesquisar suas características para facilitar sua criação, uso, conservação e transmissão. Assim, o objetivo deste estudo é identificar a metodologia de trabalho nas atividades associadas ao projeto e fabricação de helicópteros, bem como a gestão do conhecimento concebido e sua evolução ao longo do tempo em uma empresa tomada como estudo de caso. As práticas e papéis desempenhados por cada um dos atores da empresa também são aspectos significativos para a análise do caso. Utilizou-se a análise técnica de todos os helicópteros desenvolvidos pela empresa Cicaré S.A., que em particular não teve registo de atividades ou demonstrações financeiras ao longo do tempo. Além disso, entrevistas com pessoas ligadas ao atual escritório e sua história têm sido fundamentais. Cicaré S.A. é uma empresa aeronáutica argentina dedicada ao desenvolvimento e fabricação de helicópteros reconhecidos internacionalmente. É considerada referência no setor pelas inovações que desenvolveu neste tipo de aeronave, e hoje em dia é utilizada no mundo inteiro. O primeiro vôo vertical na América do Sul, o desenvolvimento de um dos primeiros helicópteros ultraleves e um simulador de vôo real controlado, são algumas das inovações que têm destacado a empresa. Desta forma, o presente estudo conseguiu identificar na empresa a coexistência de duas metodologias de design e inovação aplicadas à tecnologia aeronáutica. Por um lado, existe um departamento de produção que, utilizando metodologia de engenharia padrão (Mott, 1995), produz helicópteros em série e, por outro lado, um departamento de pesquisa e desenvolvimento que aplica a metodologia de análise de protótipos (Gero, 1990) em busca de inovações e o melhoramento de seus produtos.

Palavras-chave: Aeronáutica; Conhecimento; Design; Helicóptero; Engenharia; Protótipo. 


\section{Introducción}

En una empresa de tecnologías complejas como las de la industria aeronáutica, área en que el conocimiento aplicado proviene de campos variados de la ciencia y la tecnología, la gestión de dicho conocimiento es altamente compleja. Más aún, el conocimiento generado a diario por las tareas relacionadas con el diseño y la fabricación tiene características y particularidades que dificultan su administración.

El caso seleccionado para el análisis de esta problemática es el de Cicaré S.A., una empresa argentina de alta tecnología dedicada al desarrollo y la fabricación de helicópteros livianos, con una trayectoria de más de sesenta años. Esta empresa es considerada un referente mundial por las innovaciones que ha desarrollado y usado en este tipo de aeronaves. A lo largo de su historia, ha incursionado en el desarrollo de helicópteros con distintos tipos de estructuras y mecanismos. Sus conceptos de diseño han sido adoptados en otras partes del mundo a aeronaves de variadas performances en vuelo y de distintas motorizaciones, tamaños y sistemas de comandos. Sus diseños han puesto a Cicaré en el centro de atención de esta industria, en particular por lograr innovaciones de alto impacto mundial. El primer vuelo vertical en Sudamérica, el desarrollo de uno de los primeros ultralivianos, el sistema de rotor de vástagos y el primer simulador de vuelo vertical controlado en el mundo, son algunas de las innovaciones que han destacado a la firma en el campo tecnológico.

Sin embargo, el centro de atención de este estudio de caso es su gestión de conocimiento, partiendo de su particular método de diseño y desarrollo tecnológico. Desde sus inicios y en sus primeros desarrollos tecnológicos, la empresa Cicaré no contó con ingenieros especialistas ni realizó consultas a laboratorios de investigación y desarrollo de universidades o centros de investigación científicos. Desde sus comienzos, la base de Cicaré fue el diseño mecánico a cargo de un proyectista que alcanzó una formación formal de nivel primario, la cual se sumó a sus habilidades de herrero y metalúrgico. Este diseñador aplicó sus ideas e inició sus construcciones de manera artesanal. El responsable del proceso de diseño, desde 1961 hasta el día de hoy, ha sido Augusto Cicaré. La empresa cuenta hoy con su propia planta fabril ubicada en Saladillo, provincia de Buenos Aires, y se compone de un plantel de empleados en gran parte familiar al diseñador. El agregado de cálculo analítico, diseño industrial avanzado, nuevas tecnologías, y demás características de la industria aeronáutica, ha sido particularmente adaptado al método original artesanal de diseño.

Cabe aclarar que aquí se entenderá por gestión del conocimiento tecnológico el conjunto de actividades tales como el registro, la conservación y la aplicación de los conocimientos generados o adoptados en el proceso de diseño, a medida que el mismo evoluciona de un producto tecnológico a otro. Este concepto es significativo según el planteamiento de Nonaka (1991), quien describe la empresa tecnológica como una fuente creadora de conocimiento.

El presente estudio de caso puede ser enmarcado como un ejemplo de generación de conocimiento a partir de proyectos de tecnología compleja. Particularmente, se expone un caso en el que la tecnología se ha desarrollado en principio y ha evolucionado con cierta carencia de bases científicas formales. En esta evolución, no solamente ha alcanzado resultados valorados en la industria, sino que ha creado innovaciones aplicadas mundialmente en la industria aeronáutica. Se analizará aquí cómo la evolución del diseño a partir de los prototipos en la empresa Cicaré se ha ido retroalimentando de manera continua, y cómo el diseño se constituyó en la fuente central del conocimiento tecnológico para la firma.

En primer lugar, se plantea un análisis de estado de la cuestión y marco teórico. Allí se establecen los conceptos y argumentos de diferentes autores respecto a las temáticas centrales del estudio: el diseño, los prototipos y el conocimiento tecnológico. En segundo lugar, se desarrolla en diferentes fases cronológicas la historia de la empresa y la evolución de sus modelos de vuelo. Finalmente, se proponen conclusiones que contrastan los conceptos analizados en el estado de la cuestión y el marco teórico con los eventos desarrollados durante las fases. 


\section{Estado de la cuestión y marco teórico}

En este apartado se realiza un breve estado de la cuestión del debate sobre la generación y gestión del conocimiento tecnológico y se presentan las categorías conceptuales a emplear en el análisis del caso. Cabe aclarar que, por un lado, no existe una teoría sistemática y consolidada sobre el conocimiento tecnológico y que, por otro lado, no son abundantes los desarrollos teóricos sobre ese tipo de conocimiento en general, menos aún en países semiperiféricos como Argentina. Por lo tanto, para el análisis de este caso se realizó una selección de los abordajes y teorías más representativos de ese campo de estudios.

Ciapuscio (1996) sostiene que todo proceso cognitivo, en la tecnología, se genera por la adaptación de medios a una función preconcebida. Esta expresión se refiere al diseño ingenieril como actividad central para el tecnólogo. El diseño parte de una concepción en la mente del diseñador que se traslada a una configuración detallada a fin de ser concebido. Luego de ser plasmado en croquis, planos, o diferentes medios de transferencia, el diseño se convierte en artefacto, dispositivo o mecanismo mediante la técnica.

Se destaca la manera de ver la tecnología como el empleo del diseño para, a partir de las ideas, obtener soluciones mediante la técnica. Esto puede entenderse como un proceso básico del diseño ingenieril, según Vincenti (1990). A través de análisis de retroalimentación, puede obtenerse un diseño nuevo partiendo de la interpretación de un artefacto o mecanismo ya concebido. La tecnología posee, al igual que la ciencia, una capacidad de autoabastecerse de conocimientos; esto es, evaluar el comportamiento de los artefactos o dispositivos mediante ensayos y modificarlos para obtener mejoras; básicamente, una evolución de diseño.

Introduciéndose en la cuestión del conocimiento tecnológico, McGinn (1991) asocia este tipo de conocimiento con una gran carga de pensamiento no verbal. Menciona que una cantidad de expresiones de este pensamiento son clasificaciones del conocimiento tácito tecnológico, que no puede ser archivado. McGinn menciona que los tecnólogos cuentan con observaciones directas y experimentos de primera mano, relacionados con la técnica y el oficio. Hace un paso por métodos experimentales, concebidos como un agregado formador de pensamientos. Por último, destaca entre los anteriores un tipo de conocimiento con fuente elusiva, de inspiración intuitiva e imaginativa, propio de los ingenieros diseñadores y contrastado con el de los artistas. McGinn destaca este último tipo de conocimiento de naturaleza tácita, implícita y difícil de codificar.

En este sentido, se destaca el trabajo clásico sobre conocimiento tácito de Polanyi (1962), sobre cosas que se pueden saber, pero no se pueden explicar. Uno de los ejemplos habitualmente utilizados para ilustrar el conocimiento tácito es la habilidad de andar en bicicleta. Dicho conocimiento puede ser transmitido entre personas, aunque no mediante instrucciones explícitas o verbales, sino a través de diferentes acciones, como compartir pruebas, mostrar movimientos, corregir intentos, etc. Polanyi ha denominado a estos mecanismos de transferencia de conocimiento tácito "definiciones ostensivas, las cuales [...] siempre ocultan una brecha [gap] que tiene que ser saldada por el aprendiz con un esfuerzo de su propia inteligencia" (Polanyi, 1962, p. 602).

Los estudios sobre el conocimiento tácito suelen contrastarlo con el conocimiento explícito; es decir, el conocimiento que puede ser plasmado de manera detallada en diversos documentos. Mackenzie (1996) plantea una visión alternativa de la ciencia. Sostiene que esta se compone de un conjunto de conocimientos explícitos y conocimientos tácitos. El conocimiento explícito se refiere a todo aprendizaje capaz de ser representado por la escritura o los símbolos, que puede ser archivado en documentos. Además, el conocimiento científico cuenta con un componente tácito, del que ya se ha hablado anteriormente. El autor destaca que, por sus características, el conocimiento explícito no puede ser perdido, mientras que el conocimiento tácito sí puede desaparecer, si no es transferido entre personas de manera correcta. De esta forma, al contar con componentes explícitos que se pueden documentar, y componentes tácitos compartidos por personas, el conocimiento se convierte en avances a medida que 
va siendo ampliado. Los avances son nuevos estados del arte que difícilmente pueden ser perdidos u olvidados. Sería necesaria toda una generación de personas que no practiquen la experimentación para perder los componentes tácitos del conocimiento y, con ellos, el avance tecnológico.

En su publicación Knowing Machines: Essays on Technical Change (1996), Mackenzie ejemplifica esta visión alternativa de la ciencia a partir del análisis de caso del desarrollo de armas nucleares, preguntándose si existe la posibilidad de desaprender a construirlas:

Supongamos que la visión alternativa de la ciencia era cierta para el caso de las armas nucleares. En particular, que el conocimiento específico, local y tácito, era crucial para su diseño y producción. Entonces habría un sentido en el cual el conocimiento relevante podría ser des-aprendido, y estas armas podrían ser des-inventadas. Si hubiera un hiato suficientemente extenso en el tiempo (por ejemplo, dos generaciones) sin su producción, ese conocimiento tácito podría efectivamente desaparecer. Sin embargo, las armas nucleares aún podrían ser recreadas, pero no con la simple copia de viejos artefactos, diagramas e instrucciones explícitas remanentes. En cierto sentido, deberían ser reinventadas. (Mackenzie, 1996, p. 217)

Acerca de los tipos de conocimientos tácitos, Collins (2010) desarrolla su clasificación. El autor señala que existen tres tipos de conocimientos tácitos. En primer lugar, se encuentra el de carácter relacional, que consiste en relaciones sociales y está radicado entre personas que se transfieren una a otra un conocimiento mediante la instrucción y el aprendizaje. En segundo lugar, existe uno de carácter somático, relacionado con las diferentes maneras de resolver situaciones mediante la acción. En tercer lugar, existe un conocimiento tácito colectivo, reconocido como aquel que conforma una construcción social, un aprendizaje adoptado por un grupo de personas que comparten este conocimiento de carácter tácito y que pueden actuar a partir de comprenderlo. Collins destaca, además, que el aprendizaje del conocimiento tácito será posible en la medida en que la experimentación sobre un desarrollo sea exitosa. Es decir, los ensayos, las pruebas, los experimentos, adquieren aquí un rol fundamental en el avance tecnológico, ya que son un medio de transferencia de componentes tácitos del conocimiento tecnológico.

El método principal para aplicar el conocimiento tecnológico en el desarrollo de artefactos o dispositivos es la ingeniería. El conocimiento ingenieril es la especialización del conocimiento en ciertas áreas de la ciencia. De estas especializaciones, surgen la ingeniería civil, mecánica, aeronáutica, química, electrónica, entre otras. El conocimiento ingenieril está en el centro del análisis de Vincenti (1990). En su publicación What Engineers Know and How They Know It, el autor destaca un comportamiento evolutivo en el desarrollo de aeronaves a medida que van siendo fabricadas, probadas, corregidas y nuevamente fabricadas. Incluso establece una comparación con la evolución de las especies naturales.

Ampliando el estudio sobre el conocimiento en la ingeniería, pueden ser comparados los abordajes de Vincenti con Petroski (1992). En su publicación, To Engineer is Human, Petroski se ocupa del rol que reconocidas fallas de la ingeniería (incluso algunas catastróficas) han desempeñado en posteriores diseños eficaces. De la misma manera que Vincenti, plantea la observación de problemas ocurridos en artefactos en funcionamiento como punto de partida del diseño.

La palabra diseño proviene del latín "designare", que significa señalar o marcar. En el manual de Norton (2000) para estudiantes de ingeniería, Diseño de Maquinaria, se define la ingeniería de diseño como "el proceso de aplicar diversas técnicas y principios científicos con el objeto de definir un dispositivo, un proceso o un sistema con detalles suficientes que permitan su realización" (Norton, 2000, p. 38). Según esto, se puede interpretar que cada especialidad de diseño, como por ejemplo la mecánica y la aeronáutica, mantiene métodos que le son propios.

El método de diseño tecnológico ha sido abordado por distintos autores a lo largo de la historia. Muchos de ellos han coincidido en partes fundamentales que han logrado ser de alguna manera una base 
consensuada y estandarizada en la industria, incluso en distintas orientaciones de la ingeniería como la mecánica y la aeronáutica. Esto está detallado en el manual de diseño de Mott (1995), en el que se destacan tres instancias:

- Definiciones de requerimientos: identificar los requerimientos del usuario y definir las funciones del dispositivo.

- Creación de conceptos: proponer conceptos de diseño alternativos, evaluar propuestas y validar conceptos con base en criterios definidos.

- Diseño de detalle: selección de concepto de diseño óptimo y desarrollo de detalle final.

Las funciones señalan lo que debe hacer el aparato, por ejemplo, la capacidad de volar para una aeronave. Los requerimientos de diseño son por lo general declaraciones cuantitativas sobre los valores esperados de funcionamiento (potencia, autonomía, velocidad, etc.). Finalmente, los criterios de evaluación del diseño de detalle se centran en características cualitativas pretendidas con el diseño.

Hasta aquí es fácil apreciar que la mayoría de los autores, más allá del concepto que cada uno tenga respecto del proceso de producción de tecnología, coinciden en que la ingeniería requiere de un proceso previo a la fabricación. Es decir, antes de fabricar un modelo es necesario realizar cierto trabajo, ya sea diseño conceptual, planimetría, análisis, interpretación de requerimientos, etc. Sin embargo, es un hecho en la práctica, sobre todo en la producción de tecnologías complejas, que surgen tempranas fabricaciones de modelos que ayudan a los diseñadores a comprender el funcionamiento del producto que más tarde obtendrán sin necesidad de llegar hasta la instancia de fabricación definitiva del mismo. Esto es lo que se conoce como prototipos: ejemplares similares, comparables o iguales al producto final que se fabrican generalmente con el fin de ser someteidos a pruebas.

Con respecto a esta valoración de los aspectos teóricos y experimentales, se expresa Peter Galison en uno de los capítulos de la publicación de Mario Biagioli, The Science Studies Reader (Galison, 1999). Allí,
Galison se refiere a lo que él denomina "la zona de negociación". Esta es la instancia de un desarrollo o una investigación en la que teóricos y experimentadores tratan de comprender un fenómeno o comprobar una hipótesis, de manera positivista o antipositivista, reduciendo el fenómeno a una teoría o a un experimento, dependiendo el caso. De la misma manera, se puede analizar este comportamiento en la ingeniería. Aquí, la zona de negociación se traduce a la etapa en la que los teóricos y experimentadores tratan de comprender el funcionamiento de un artefacto a través de análisis o a través de ensayos de prototipos, respectivamente. Esto es, se pueden desarrollar distintas tecnologías o bien con un mayor enfoque teórico, o bien con una predominancia del criterio experimental; el criterio a seguir dependerá del tipo de tecnología o del proyecto específico.

Quienes se inclinan mayormente por la experimentación como base de la generación de conocimientos, tienden a fabricar tempranamente estructuras o mecanismos para ensayar durante el desarrollo. Esto puede ser parte de lo que será el producto final, o directamente un modelo igual al que se busca obtener para someterlo a evaluación; esto es, lo que se denomina prototipo.

Un prototipo es un primer ejemplar de un artefacto empleado para realizar ensayos, calificar su uso en el contexto en el que funcionará, aplicarle correcciones y evaluar su funcionamiento. Un prototipo calificado y aceptado puede servir además como referencia para fabricar una serie. Acerca de esto, Gero (1990) sostiene que el propósito de un diseñador es transformar una función determinada en una descripción de diseño que detalla cómo será el aparato que cumple la función. Por ejemplo, diseñando ventanas, las funciones pueden ser permitir el ingreso de luz exterior a un cuarto, regular la ventilación o poder ver al exterior. La descripción del diseño será un texto, croquis o plano que detalle las dimensiones de la ventana a construir. Finalmente, el artefacto será la ventana. De esta manera, se podrá obtener un primer ejemplar prototipo que tendrá un comportamiento particular. Sin embargo, se deberá realizar una comparación entre ese comportamiento del prototipo y el comportamiento esperado 
por el diseñador. Esta comparación no es más que la evaluación del prototipo. A partir de la evaluación se realizarán modificaciones al prototipo hasta que este se comporte de una manera más adecuada al comportamiento esperado. Gero denomina a este proceso "síntesis".

Cuando el prototipo se encuentra sintetizado, tiene un comportamiento propio que además es el que el diseñador esperaba en un principio. Cuando el comportamiento esperado o deseado es inalcanzable, o difícilmente alcanzable por el prototipo, el diseñador puede modificar su requerimiento; este proceso es denominado "reformulación". Finalmente, Gero concluye que existen dos grandes aspectos en la construcción de prototipos:

- La representación del diseño en un prototipo, es decir, modelar un prototipo que sea una fiel reproducción del diseño esperado.

- La apropiación de conocimiento a través del prototipo, que significa obtener conocimientos a partir de la evaluación del prototipo para reformular el diseño o generar uno nuevo.

Este segundo aspecto, que incluye la posibilidad de obtener un funcionamiento del prototipo distinto al esperado, puede llevar al descubrimiento de una innovación. Es decir, se obtiene un comportamiento del prototipo distinto al buscado por el diseñador, pero este puede ser valorable. Si quiere, el diseñador puede apropiarse de estos conocimientos para transformarlos en innovaciones.

\section{Metodología de estudio}

Los siguientes acápites serán detallados de acuerdo a una clasificación por fases. Estas consisten en periodos caracterizados por eventos que generaron cambios en metodologías de diseño particulares sobre modelos concretos desarrollados por la empresa. Con base en esto se ha establecido una metodología de estudio segmentada. Para cada fase de estudio se han realizado las siguientes tareas de investigación:
- Entrevistas: se han realizado entrevistas a diferentes actores, como empleados, exempleados, asesores, proveedores y clientes de la empresa.

- Análisis de tecnologías: se han analizado las características técnicas y tecnológicas de los helicópteros desarrollados.

- Consulta bibliográfica: se ha indagado en bibliografía que conforme un marco teórico para las investigaciones realizadas y permita un análisis detallado de conceptos e hipótesis. Finalmente, con el análisis de bibliografía histórica se comprende el contexto político, social y económico de cada fase, en contraste a los avances de la empresa.

Más allá de las investigaciones de cada fase, se analizaron las relaciones interpersonales, los desarrollos de tareas de producción, la administración de la documentación, la disposición de prototipos, entre otras características de trabajo particulares, mediante una visita a las instalaciones de la fábrica.

\section{Fase I. El desafío de volar. Los modelos CH-1 y CH-2 (1956-1963)}

A principios de la década de 1950, Augusto Cicaré se desempeñaba como ayudante en la metalúrgica de su padre en la localidad de Polvaredas, un pequeño poblado de la provincia de Buenos Aires. Sus conocimientos técnicos provenían de sus labores en herrería, soldadura y mantenimiento mecánico aplicado a maquinaria agrícola típica del interior de Buenos Aires.

Por aquel entonces era frecuente que los talleres mecánicos y la gente aficionada a la mecánica estuvieran suscritos a los ejemplares de la revista Mecánica Popular, que recopilaba y publicaba artículos sobre avances tecnológicos en el área de la mecánica en todo el mundo. En una de estas publicaciones, Cicaré había apreciado años atrás la figura de un artefacto que causó su asombro. En una breve nota, se detallaban exposiciones y demostraciones del inventor Sikorsky 
y su aeronave de vuelo vertical: el helicóptero. Aquella imagen, que había permanecido durante años en la memoria del joven Cicaré, se convirtió en una idea de desarrollo cuando alcanzó su adolescencia; tenía entonces 17 años. Todo el trabajo mecánico y metalúrgico anterior a la idea de fabricar un helicóptero, se puede considerar que le dio a Augusto Cicaré aquellos "medios preconcebidos" mencionados por Ciapuscio (1996), los que luego el diseñador adaptó para obtener soluciones aeronáuticas.

El mayor conflicto del proceso de desarrollo de una aeronave fue la falta de conocimiento tecnológico aeronáutico. Cicaré contaba solo con sus estudios primarios, sus habilidades en el taller mecánico y una fotografía extraída de una revista en la que se observaba un helicóptero Sikorsky experimental con su creador de pie a un costado. Sabía que en distintas partes del mundo esta tecnología había sido desarrollada, pero no tenía información sobre ella. Este conflicto aparece en la reflexión de Norton (2000), quien plantea que evidentemente existe un conocimiento tecnológico específico de la aeronáutica que no es fácilmente dominable por personas especialistas en otras áreas similares, como la mecánica general o la metalúrgica.

La idea inicial de Cicaré era fabricar de manera artesanal un chasis monoplaza similar al que podía observarse en aquella fotografía. Para esto tomó como medidas y formas geométricas las comparaciones entre el tamaño de la aeronave, tal como se apreciaba en la imagen, y el propio diseñador parado a su lado, considerado patrón de referencia. El ejercicio resultó en una estructura alcanzada con soluciones mecánicas poco ortodoxas en aeronáutica. Esto se puede analizar en términos de lo que Layton (1971) denomina "ideología de diseño”. Según este concepto, el único objetivo de la obtención del producto es la producción en sí misma, sin que existan requerimientos exigidos por un tercero.

Desde el punto de vista del proceso, los requerimientos que el diseñador registró para comenzar su diseño provinieron de su propia deducción:

- El aparato se sustenta en el aire por el empuje generado por palas giratorias.
- El dispositivo debe contar con un habitáculo para su piloto.

- Debe existir un par extra de palas que gire en sentido contrario a las principales, o un rotor de cola, para impedir el giro descontrolado del habitáculo.

- Debe existir un sistema de comandos que permita elevar, descender, avanzar y dirigir el artefacto según la decisión de su piloto.

Es claro que esta falta de vínculo entre el diseñador y los avances alcanzados en la industria de aquel entonces en materia de helicópteros conformaba de alguna manera ese hiato de conocimiento que menciona Mackenzie (1996). Esto imposibilitaba que Cicaré continuara con el desarrollo de helicópteros ya existentes, pues carecía del conocimiento explícito y tácito sobre este tipo de aeronaves y la aeronáutica en general. De manera que, como señala Mackenzie, la única alternativa que tenía el diseñador para desarrollar un helicóptero era reinventarlo.

Avanzando en el proyecto, Cicaré se dedicó al diseño y fabricación del rotor de la aeronave (sin dudas, el más complejo de los sistemas para este tipo de artefactos). El rotor de un helicóptero es un mecanismo que transforma la potencia del motor en el movimiento de dos o más palas diseñadas con perfil alar, lo que le da a la aeronave la sustentación. El sistema contrarrotativo fue diseñado e instalado sobre el artefacto. Este contaba con una estructura de paralelogramo deformable que permitía mover el centro de gravedad de la aeronave. De esta forma se lograban las maniobras durante el vuelo, por medio de un sistema de palancas que accionaba este mecanismo. La motorización de una motocicleta fue adaptada a la nave.

En cuanto al diseño de aspas, el diseñador había observado en fotografías que el cuerpo era de terminación biconvexa. Una visita al aeroclub de Saladillo para observar las hélices de los aviones bastó para fabricar palas de perfil similar con la ayuda de un proveedor de modelado en madera. El escalado dimensional fue calculado a través de la observación de la fotografía. A partir de ella dedujo que las palas tenían el ancho de una cabeza humana y una longitud seis veces mayor 
al mismo patrón de medida. De esta manera, todos los mecanismos del helicóptero fueron completados para 1956.

En 1957, Augusto Cicaré inició una serie de ensayos de su aeronave prototipo. Aunque no realizó un registro de las pruebas, se sabe que con las primeras se pudo comprobar que las aspas giraban de manera correcta, pero la baja potencia hizo evidente que no se lograría el vuelo vertical. Así fue que Cicaré se avocó completamente al diseño y construcción de un nuevo motor. El resultado fue un motor artesanal con block fundido en aluminio, de cuatro tiempos, dos cilindros opuestos y $1.050 \mathrm{~cm}^{3}$ de cilindrada, que ofrecía una potencia de $30 \mathrm{Hp}$.

Los ensayos con el nuevo motor demostraron que la aeronave lograba despegarse del piso, aunque luego de unos segundos caía por su propio peso, debido a la pérdida de inercia del motor. En este ensayo, Cicaré observó que una aceleración en la velocidad angular del rotor generaba un incremento en la inercia y alargaba el tiempo de vuelo del helicóptero. Sin embargo, nunca pudo superar el minuto de acción. Decidió repetir el ensayo sin el tripulante a bordo: accionó los comandos parado sobre el piso a un costado de la nave y mantuvo el equilibrio de la misma con la ayuda de dos personas más (esto demuestra a las claras los rústicos métodos de ensayo empleados). Estas pruebas le permitieron mejorar notablemente el sistema de comandos. Aquí se puede apreciar la relación entre el aprendizaje y la experimentación de la que habla Collins (2010). Cicaré recurrió a la experimentación del vuelo para comprender los fenómenos físicos que lo condicionan.

Finalmente, hacia 1959 aproximadamente, una falla en la coordinación de las tres personas que ejecutaban el ensayo derivó en el descontrol del helicóptero. Este se soltó de las manos de quienes lo sostenían, voló y se estrelló a pocos metros con el piso. El primer prototipo, llamado Cicaré $\mathrm{CH}-1$ había sido completamente destruido. Hay que destacar que existió una primera evolución en la motorización debido a la falta de potencia inicial, la cual permitió acercar al diseño al vuelto vertical. En segundo lugar, la evolución provocó seguidamente una pérdida de control y una fuerte falla en el método de diseño. Estas dos apreciaciones se sustentan en la evolución de modelos (Vincenti, 1990) y las fallas desencadenantes de cambios de diseño (Petroski, 1992).

El diseño de la segunda unidad del CH-1 Comenzó a principios de la década de 1960 . Se repitió la construcción anterior, aunque se emplearon algunas modificaciones: una ampliación de la cabina, la construcción totalmente metálica de las aspas y la mejora en el sistema de paralelogramo deformable que permitía el avance del helicóptero. En cuanto a motorización, Cicaré diseñó y fabricó un nuevo motor de dimensiones duplicadas con respecto al anterior. Este ofrecía una potencia de $60 \mathrm{Hp}$ para paliar las pérdidas de inercia observadas en los ensayos del primer prototipo $\mathrm{CH}-1$.

Los ensayos del nuevo $\mathrm{CH}-1$ fueron llevados a cabo por Cicaré sobre un precario banco de pruebas. Simplemente se anclaba el helicóptero al piso con cuatro cadenas que le impedían tomar una altura considerable de vuelo. Esto podría ocasionar un accidente, teniendo en cuenta que el diseñador no tenía la capacidad de pilotear con total control la aeronave. Los ensayos fueron exitosos: pudo alcanzar más de cinco horas de vuelo y aprendió además a controlar el helicóptero. Esto le permitiría salir a campo abierto para realizar nuevos ensayos de vuelo sin las cadenas de anclaje (figura 1).

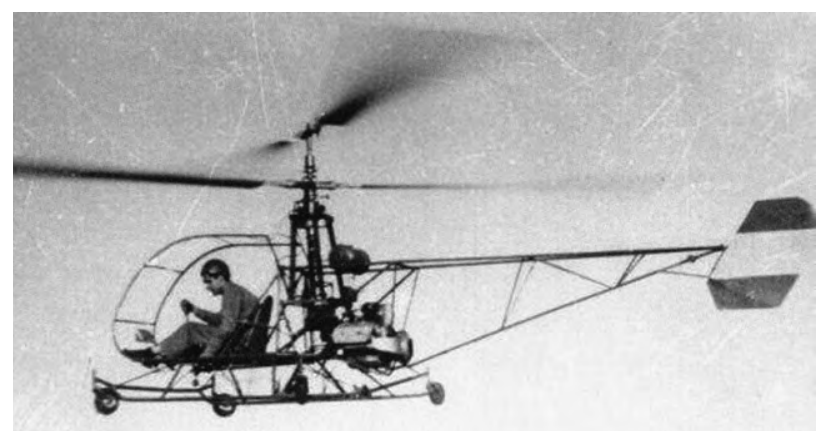

Figura 1. Vuelo de ensayo del modelo $\mathrm{CH}-1$ a cargo de Augusto Cicaré. Polvaredas, 1960

Fuente: Cicaré S.A. (2016). 
Después de completar unas quince horas de pruebas, los vuelos en campo abierto mostraron muchas fallas en el sistema de comando. En conclusión, el vuelo de la aeronave dependía mucho de las condiciones de viento, aunque los ensayos demostraban que el diseño era viable y funcional. Se puede hacer una conexión aquí con la comparación entre el comportamiento real y el comportamiento esperado mencionada por Gero (1990). Se cuenta con un prototipo que cumple las funciones de vuelo vertical que el diseñador buscaba, sin embargo, el diseño específico no resulta valorable.

Durante una exposición industrial realizada en la localidad de Saladillo, Augusto Cicaré conoció al fotógrafo Santiago González Valdez, fotógrafo oficial de la Presidencia de la Nación Argentina. Interesado por el desarrollo tecnológico de Cicaré, González Valdez utilizó sus contactos personales para proveer al diseñador de un paquete de libros y revistas sobre aeronáutica. Le ofreció también una ayuda económica para colaborar con los gastos del nuevo prototipo que Cicaré estaba comenzando a desarrollar, el CH-2. Aparte de aquel acercamiento, González Valdez informó a un oficial de la Fuerza Aérea Argentina sobre los prototipos de aeronaves Cicaré; se trataba de Ildelfonso Durana, quien además era técnico mecánico y piloto de helicópteros.

En 1963, Ildelfonso Durana concedió a Augusto Cicaré una visita a la Base Oficial de Aviación Civil (BOAC) en la localidad de Cuartel v, partido de Moreno. Esta visita fue más que importante para Cicaré, quien pudo observar por primera vez un helicóptero de construcción industrial empleado en vuelos profesionales.

Luego de obtener tantos avances en su conocimiento tecnológico, Cicaré emprendió el diseño del segundo prototipo, $\mathrm{CH}-2$, el cual proyectaba con soluciones aeronáuticas más avanzadas y el empleo de materiales de uso corriente en estructuras de aeronaves. Por aquel entonces, y por iniciativa de Santiago González Valdez, se formó una agrupación de interesados en obtener financiamiento económico para los desarrollos de Cicaré mediante eventos benéficos.

En 1964, comenzó la construcción del Cicaré CH-2, un helicóptero biplaza lado a lado con cabina cerrada, tren de aterrizaje con patines, un motor de aeronaves
Lycoming 0-360 provisto por la Fuerza Aérea, rotor principal de tres palas y rotor bipala de cola. El mecanismo de transmisión a la cola fue diseñado y construido por Cicaré, quien empleó correas y una caja de engranajes. La estructura principal del helicóptero constaba de un reticulado también ideado por Cicaré.

Vale la pena observar lo sucedido con el diseño de rotor. En primera instancia, Cicaré optó por basarse en los diseños de Sikorsky, aunque no pudo conseguir que ellos fueran efectivos. Decidió entonces descartarlos y crear un nuevo sistema según su imaginación. Se puede volver a destacar en este episodio la interpretación primitiva de un problema. El rotor propio constaba de una torreta de accionamiento hidráulico mediante pistones y resortes que permitían direccionar las aspas para maniobrar la aeronave.

En 1965 finalizó la construcción del cH-2. Cicaré inició su campaña de ensayos, para lo cual diseñó y construyó un nuevo e innovador banco de pruebas sin precedentes. El banco contaba con una estructura anclada al piso que capturaba el helicóptero para limitar su altura de vuelo, pero que permitía una libertad suficiente para realizar todo tipo de maniobras de control. Los ensayos en aquel novedoso banco de pruebas fueron exitosos: el vuelo del helicóptero $\mathrm{CH}$-2 era correcto y su maniobrabilidad era muy eficaz. Esta incursión en el estudio de materiales, definitivamente más rudimentarios y elemental que los ensayos de vuelo, puede ser analizada desde la perspectiva de Galison (1999). El diseñador comienza a estudiar fenómenos físicos como el efecto giroscópico. A pesar de su falta de conocimiento en ciencias básicas, contaba aquí, sin dudas, con sus observaciones y experimentos de primera mano, como especifica McGinn (1991).

\section{Fase II. De Polvaredas a Oshkosh. Los modelos CH-3 Colibrí, CH-4, CH-5 у Сн-6 (1963-1990)}

En 1972, Augusto Cicaré formó la sociedad Cicaré Aeronáutica Sociedad en Comandita, junto a Idelfonso 
Durana y Antonio Raul Mantel, dos comodoros retirados de la Fuerza Aérea Argentina. La idea inicial era elaborary presentar al Consejo de la Industria Aeronáutica un plan de negocios para la fabricación de un helicóptero producto de la evolución del CH-2. Dicha institución estaba dedicada precisamente a la promoción y financiación de proyectos aeronáuticos. Con aquella iniciativa obtuvieron una subvención económica en 1973.

Augusto Cicaré y sus socios se disponían a diseñar y construir un nuevo helicóptero, esta vez con financiación del Estado. Para ello consiguieron previamente la donación de un terreno en la localidad de Saladillo, provincia de Buenos Aires. Allí era mucho más factible la instalación de un taller hangar con los servicios básicos que en Polvaredas eran difíciles de conseguir. Una vez Cicaré se radicó en la nueva locación, se dio inicio al proyecto del nuevo modelo denominado CH-3 Colibrí, nombre que dejaba en claro la idea definitiva de dedicarse a la línea de helicópteros livianos. Se trataba de un biplaza de habitáculo flexible que permitía la inclusión de una plaza extra. Tenía un rotor principal de cuatro palas y un rotor de cola bipala con motor Crysler v8. Se puede destacar que la característica evolutiva principal (Vincenti, 1990) hasta este modelo es la potencia de motor.

Un año más tarde se presentó por primera vez un modelo Cicaré a nivel internacional. Aerosaladillo Sociedad Anónima presentó el modelo Colibrí en los Estados Unidos; más exactamente, en la Convención de Vuelo de la Experimental Aircraft Association en Oshkosh (estado de Wisconsin). Allí, el modelo recibió la mirada curiosa de expertos en aeronáutica de todo el mundo y cosechó excelentes críticas, sobre todo con respecto a sus mecanismos innovadores.

A su regreso a Argentina, la Dirección Nacional de Aeronavegabilidad canceló la homologación para vuelo del Colibrí. Estos rechazos jamás pudieron ser negociados, por lo que el proyecto Colibrí vio su fin. Esto ocasionó además la disolución de Aerosaladillo Sociedad Anónima en 1978. Una nueva crisis económica en el país mantuvo a Augusto Cicaré dedicado a sus proyectos no aeronáuticos junto a sus hermanos por unos años.

Luego del diseño y construcción de un modelo CH-4, que no será detallado en este estudio, en 1985 la Fuerza Aérea Argentina mostró su interés en trabajar en conjunto con la fábrica de Saladillo. Augusto Cicaré partió de todos sus diseños anteriores y se propuso fabricar un helicóptero monoplaza bipala con rotor de cola y motorización Lycoming 0-320-A2C. Así nació el Cicaré $\mathrm{CH}-5$.

El diseño fue aceptado por la Fuerza Aérea, que le dio un plazo de dos años a Cicaré para la construcción de un prototipo para ensayos. Para ello, Cicaré comenzó con la fabricación de un banco de pruebas para ensayar las partes en desarrollo. Ese banco de pruebas se transformó básicamente en otro helicóptero de similar prototipo, y de dimensiones algo menores. Este fue denominado $\mathrm{CH}-6$.

Es menester ahondar en los detalles de aquel prototipo experimental, que representó tiempo después mucho más que un dispositivo de pruebas para la empresa. El CH-6 era, al igual que el $\mathrm{CH}-5$, un monoplaza bipala con rotor de cola, aunque con dimensiones reducidas a escala del principal, y con un motor extraído de un prototipo anterior. Lo novedoso de este aparato fue su sistema de rotor. Los rotores que emplean los helicópteros del mercado poseen un mecanismo denominado "plato oscilante", cuya función es inclinar las palas del helicóptero desde la cabina del piloto, lo que varía el empuje que ellas entregan. Aunque en todos los modelos del mercado el plato oscilante se ubicaba en la cabeza del rotor, Augusto Cicaré decidió trasladar el sistema a la parte inferior de la caja de engranajes, que distribuye la potencia entregada por el motor a los rotores principal y de cola, lo cual simplifica la fabricación del mismo. La sorpresa para todos llegó durante las primeras pruebas del prototipo. El helicóptero poseía una maniobrabilidad y sensibilidad en sus movimientos nunca antes vistas, incluso según la apreciación de pilotos acostumbrados a comandar aeronaves líderes en el mundo, como Bell o Sikorsky.

De manera inesperada, la innovación desarrollada por Cicaré estaba desviando el interés de los expertos hacia la versión reducida del $\mathrm{CH}-5$. Los expertos comenzaron a evaluar la posibilidad de adaptar el prototipo $\mathrm{CH}-6$ a una versión apta para homologación de vuelo, lo que potencialmente permitiría su fabricación en serie. La maniobrabilidad alcanzada por la 
innovación de Cicaré permitía incluso realizar acrobacias de vuelo sin precedentes en helicópteros.

En 1989, Argentina enfrentó una de sus crisis económicas y sociales más destacadas (incluso se dio la renuncia del presidente y la respectiva asunción del recientemente electo Dr. Carlos Saúl Ménem Wainer, 2018). Una vez más, la empresa recibió la noticia que tanto se repitió en su historia: el proyecto de fabricación en serie fue suspendido.

Si bien en Industrias Cicaré lamentaban la discontinuidad del proyecto principal, mantenían su esfuerzo dirigido al innovador prototipo $\mathrm{CH}-6$. Augusto Cicaré formó una nueva sociedad denominada Aeronaves Saladillo Sociedad Anónima con el fin de fabricar de manera seriada el ultraliviano.

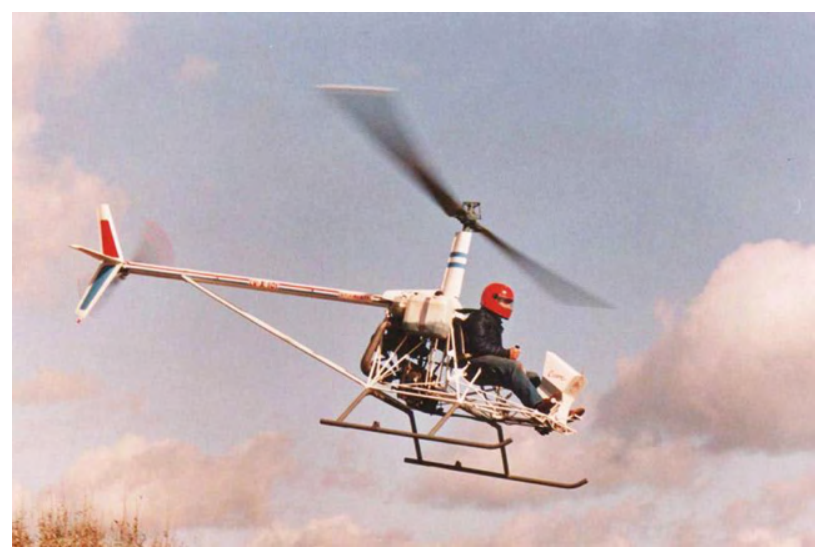

Figura 2. Ensayos de vuelo sobre el modelo $\mathrm{CH}-6$ a cargo de Augusto Cicaré. Saladillo, 1989.

Fuente: Cicaré S.A. (2016).

Las innovaciones introducidas en el modelo $\mathrm{CH}-6$ fueron del interés de muchos expertos en tecnología aeronáutica. Tal fue el caso de un piloto estadounidense y ex combatiente en Vietnam, Dennis Fetters. Fetters era dueño de una empresa dedicada a la construcción de autogiros. Gracias a un intermediario argentino conoció los diseños Cicaré, lo que lo llevó de visita a Argentina en 1990.

Fetters le propuso a Cicaré la firma de un preacuerdo para la fabricación del modelo en Estados Unidos, que se haría después de la exposición del prototipo en la Convención de la EEA (Experimental Aircraft
Association) de ese año en Oshkosh. Pocos meses después, el modelo fue exhibido y probado en la exposición norteamericana. Previo al traslado del modelo, Cicaré inició trámites de patentamiento de su sistema de rotor sin plato oscilante.

Durante la convención estadounidense, Augusto Cicaré notó que el manejo de la información de Dennis Fetters en la exposición confundía a parte de los visitantes de la muestra sobre los orígenes de tal innovación. Esta fue definitivamente el éxito de la convención en aquella edición. Por tal motivo, Cicaré decidió ampliar la patente a registro estadounidense. Después de lograr que su patente ingresara a las oficinas de propiedad intelectual norteamericana, Cicaré advirtió que una patente de características muy similares había ingresado días antes a nombre de Dennis Fetters. En ese momento, Fetters ya había fundado una empresa ad hoc denominada Revolution Helicopters para la construcción de un modelo denominado Mini 500 que se basaba en la tecnología recientemente desarrollada por Cicaré. La noticia generó una ruptura inmediata de la relación entre el inventor argentino y el empresario estadounidense al final de la convención. Además, Cicaré inició un juicio contra el estadounidense.

El diseño argentino fue todo un éxito, aunque Aeronaves Saladillo S.A. no se benefició del mismo, pues Revolution Helicopters vendió más de quinientas unidades del modelo Mini 500. Si bien el Mini 500 fue exitoso, definitivamente las características del $\mathrm{CH}-6$ que fueron novedad en la convención no fueron logradas por aquel diseño adaptado.

\section{Fase III. Un vuelo por el mercado mundial. Los modelos $\mathrm{CH}-7$ y SVH (1990-1993)}

Los inicios de la década de 1990 marcaron una transición entre la hiperinflación de la moneda de finales del gobierno del presidente Dr. Raúl Alfonsín y la convertibilidad monetaria de la gestión del presidente Dr. Carlos Ménem. A pesar de la transición, en esta época la crisis económica y social del país se mantuvo. 
En 1990, Augusto Cicaré realizó unas modificaciones mínimas al modelo $\mathrm{CH}-6$ por consejo de pilotos e idóneos en aeronáutica. Las modificaciones buscaban afianzar aún más las prestaciones de vuelo del diseño. Inmediatamente, comenzó la fabricación de un nuevo modelo optimizado que finalizó a finales de 1990 para ser ensayado en 1991. Los ensayos estuvieron a cargo del reconocido piloto de autogiros Gustavo Brea, quien ya había asesorado anteriormente a la empresa para la optimización de distintos modelos. Si bien el diseño era igual al $\mathrm{CH}-6$, con las modificaciones por motivos de seguridad de la estructura, fue renombrado $\mathrm{CH}-7$. Al igual que su antecesor, se trató de un monoplaza bipala con rotor de cola y motor Rotax 582 de $64 \mathrm{Hp}$. Es necesario notar que se incorporaron aquí, como en otros modelos, opiniones de terceros, a veces expertos, que modificaron o agregaron requerimientos de entrada al proceso de diseño, tal como lo detalla Gero (1990). Cicaré tuvo en cuenta esos requerimientos a la hora de evaluar sus construcciones.

Mientras se desarrollaba el $\mathrm{CH}-7$, Revolution Helicopters ultimaba los ensayos y la puesta a punto del modelo Mini 500, el cual presentaba muchos inconvenientes debido a la falta de asesoramiento de su creador en Argentina. Muchos fabricantes y concesionarios internacionales habían recibido referencias de la performance del $\mathrm{CH}-6$.

Un ejemplo de ello surgió de un fallido contrato para la venta de varios modelos Mini 500. El contrato fue firmado por la compañía Elisport, radicada en la ciudad italiana de Turín y a cargo del empresario aeronáutico Pierluigi Barbero. Elisport empezó a recibir reclamos por la extensión de los plazos de entrega a sus clientes europeos, lo que generó la ruptura de la representación. A través de un intermediario argentino, el empresario italiano se enteró de que el diseño original de Fetters era en realidad de un inventor argentino. Pocos meses después de la ruptura del contrato con Revolution Helicopters, Pierluigi Barbero viajó a la Argentina junto a uno de sus principales asesores técnicos para encontrarse con el creador del modelo que lo había interesado.

Luego de unas pruebas de vuelo en el $\mathrm{CH}-7$, recientemente homologado y patentado, Barbero pudo verificar la superioridad en prestaciones y la flexibilidad de maniobras que el modelo argentino mostraba, en comparación con la aeronave de Fetters. La propuesta del empresario italiano fue fabricar el modelo $\mathrm{CH}-7$ en su fábrica de Turín bajo licencia y con el apoyo de Augusto Cicaré como asesor técnico.

Luego de un tiempo de negociaciones, en marzo de 1992, Augusto Cicaré viajó a Turín. Treinta días antes del viaje, había sido despachado el prototipo $\mathrm{CH}-7$ (que además había sido adquirido por el mismo Pierluigi Barbero). Augusto Cicaré trabajó en el armado del helicóptero recientemente comprado por el gerente de Elisport y finalizó días después su puesta a punto. Días más tarde el modelo fue presentado en el Meeting Internacional de Ultralivianos en Bassano de Grappa.

Finalizada la convención, Barbero contrató a un importante diseñador industrial para que diera forma a una cabina estilizada que le dio la identidad final al producto comercializado por Elisport, denominado CH-7 Ángel. Finalmente, se firmó un acuerdo de fabricación bajo licencia entre Elisport y Augusto Cicaré, con lo que se puso en marcha la producción en serie del helicóptero en Turín.

Se distribuyeron más de 300 unidades del $\mathrm{CH}-7$ Ángel en todo el mundo. Una vez que Augusto Cicaré regresó a Argentina, la empresa Elisport, ahora convertida en Helisport S.R.L., hizo un reclamo al diseñador argentino por una falla técnica que no corresponde al modelo original y por ciertos aspectos del contrato de fabricación bajo licencia. Debido a esto, los pagos de las regalías fueron retenidos. Augusto Cicaré inició el segundo litigio contra un fabricante extranjero por pagos de cánones, que finalmente jamás pudo recibir, de los cientos de unidades vendidas en todo el mundo. Pierluigi Barbero acudió a Dennis Fetters, que aún se mantenía en conflicto judicial con Cicaré, y se basó en su patente para seguir fabricando helicópteros sin pagar regalías hasta que fueran concluidos todos los litigios internacionales.

En 1992, en Argentina se estaba dando cierta reactivación de la economía. En un escenario de ajuste fiscal, el gobierno decidió recortar todas las subvenciones para desarrollos tecnológicos. Debido a esto, la fábrica de Saladillo solo podía vender el modelo 
CH-7 a compradores particulares y privados. Se inició aquí la formación de la empresa Cicaré Helicópteros S.A., firma que sigue existiendo hasta la actualidad. En 1993, la empresa se disponía a comercializar el modelo $\mathrm{CH}-7$, que ya se vendía exitosamente en Europa y se conocía como Mini 500 en Estados Unidos. Años más tarde, a principios de la década del 2000, la empresa se basó en el diseño original $\mathrm{CH}-7$ para construir un nuevo modelo con cabina estilizada y un instrumental electrónico modernizado. Este modelo fue denominado $\mathrm{CH}-7 \mathrm{~B}$, o también $\mathrm{CH} 2000$ para las ventas hechas durante el cambio de milenio.

A principios de la década de 1990, se incorporó a las tareas de fabricación el mayor de los hijos de Augusto Cicaré, Fernando. En esta época, el joven de alrededor de quince años se interesó en formarse como piloto, además de colaborador de su padre, quien estaba al frente de Cicaré Helicópteros S.A., una empresa formal. Para el aprendizaje de vuelo, en 1993, Augusto Cicaré retomó la idea de desarrollar un simulador de vuelo optimizado, una evolución del helicóptero prisionero con cadenas empotradas al piso que le había dado a él la posibilidad autodidacta de aprender a volar en la década de 1960. Sin dudas, el diseñador apostó a la experimentación como método de aprendizaje completo (incluido el componente tácito), como lo destaca Collins (2010).

En la empresa se dieron cuenta de que el simulador les permitiría formar a potenciales clientes, por lo que se ofrecía, además del producto, la formación como piloto. Se intentaba lograr un producto accesible a clientes particulares, pero la falta de formación de vuelo era un inconveniente para ofrecer el producto mismo. La optimización del sistema ideado para la formación de Fernando Cicaré dejó un concepto de producto que muchos pilotos cercanos a la empresa recomendaron patentar y producir para su comercialización.

El proyecto que había empezado a gestarse se denominó Simulador de Vuelo de Helicóptero SVH-1, y se eligió el modelo $\mathrm{CH}-6$ como nave cautiva. Al principio, solamente se permitía el control de acelerador, paso colectivo y pedales. Prontamente se decidió evolucionar el diseño y producir un SVH-2 (hacia fines de 1993). Este diseño también contaba con una nave
$\mathrm{CH}-6$, ahora cautiva a una pista compuesta por una larga barra estructural que permitía el giro y el traslado del aparato en vuelo. El diseño ya mostraba resultados óptimos. De todas maneras, se decidió estandarizar la fabricaciónempleandocomohelicópterocautivounmodelo $\mathrm{CH}-7$. Finalmente, el diseño de detalle fue denominado $\mathrm{SVH}-3$. Este cuenta con un monoplaza $\mathrm{CH}-7$ sujetado por dos columnas estructurales de extensión variable a sus lados, lo que permite el ascenso y descenso de la aeronave mediante un sistema neumático.

A principios de 1995, el producto fue completado y representantes de la Escuela Federal de Aviación, dependiente del Cuerpo Federal de Aviación de la Policía Federal Argentina, acudieron a la fábrica de Saladillo para supervisar el simulador. Un año más tarde, los cursos oficiales de pilotos ya contaban con la tecnología Cicaré para formar a sus alumnos. Poco tiempo después de comenzar la instrucción de pilotos, la escuela adquirió dos unidades más y las repartió en su dependencia de Campo de Mayo.

En el 2001, el simulador, que ya había sido presentado en varias exposiciones, recibió la certificación por parte de la Federal Aviation Administration de Estados Unidos. Esto permitía el empleo de la tecnología en la formación de pilotos norteamericanos. Años más tarde, el simulador ya era empleado en instrucción de vuelo en Argentina, Estados Unidos, Brasil, Inglaterra, Suiza, Australia, Chile, Austria y Marruecos. En la actualidad, se ofrece como producto una última evolución de performance denominado SvH-4 (figura 3).

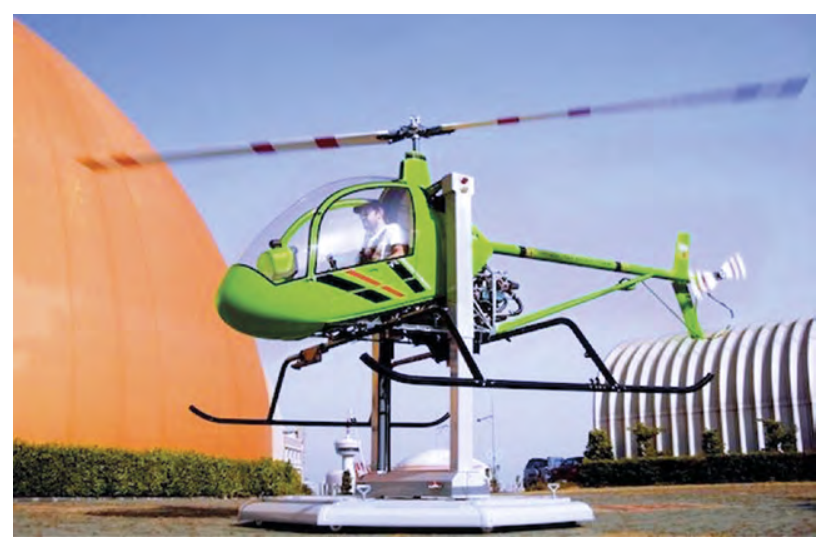

Figura 3. Modelo SVH-4 en pruebas de instrucción en el 2002. Fuente: Cicaré S.A. (2016). 


\section{Fase IV: La industria Cicaré. Los modelos CH-11 y CH-12 (1993-2001)}

Con la experiencia en el diseño y la construcción de los modelos $\mathrm{CH}-6$ y $\mathrm{CH}-7$, la empresa pudo capitalizar avances de un prototipo a otro. Para ese momento, habían surgido innovaciones que se aplicaban de manera general en toda la industria aeronáutica a nivel mundial.

En la búsqueda de nuevas alternativas y variaciones para ampliar el mercado del modelo $\mathrm{CH}-7$, Augusto Cicaré comenzó una campaña de experimentación sobre el mismo. La primera aplicación que suponía una mejora era la ampliación de la potencia de vuelo; agregar un motor y convertir al helicóptero liviano en bimotor suponía un producto de alto potencial, pues permitiría el vuelo de un pasajero acompañante o alguna carga útil definida por el cliente. Este fue el principio de desarrollo del modelo $\mathrm{CH}-8$ que, sin embargo, mostró algunas dificultades de vuelo. La actividad desarrollada en este periodo no era más que la asignación de nuevas funciones a cumplir en el proceso de diseño (Gero, 1990).

Durante 1997, comenzó la construcción de una nueva aeronave basada en el diseño $\mathrm{CH}-7$. Las diferencias eran la ausencia de rotor de cola y el agregado del sistema de rotores coaxiales. Se seleccionó una motorización ya conocida por Cicaré, el Rotax 618 de $75 \mathrm{Hp}$. Esto dio origen a un modelo interesante por su tamaño y peso reducido, con respecto a su antencesor, el $\mathrm{CH}-7$. Además, el modelo tenía una gran capacidad de carga, estimada en un $15 \%$ mayor que el anterior modelo. La denominación para el modelo fue $\mathrm{CH}-11 \mathrm{C}$. El agregado de una letra a la nomenclatura típica de Cicaré señala su cualidad de contrarrotativo.

El helicóptero cuenta con una buena maniobrabilidad y reducido tamaño. Esto llevó a incluir en su diseño la posibilidad de agregar un sistema de control remoto, con el que se podría transformar la nave en UAV (Unmaned Aerial Vehicle -Vehículo No Transportado-). Esto abrió un nuevo mercado en competencia con los actuales modelos de tipo dron, aunque, en el caso de Cicaré, de superior capacidad de carga.

\section{Fase v: Cicaré y el Estado Argentino. El modelo CH-14 Aguilucho (2001-2015)}

Luego de la crisis socioeconómica del 2001, que obligó a la renuncia del entonces presidente Dr. Fernando De La Rúa, y después de varios cambios de presidentes provisionales y la final posesión del Dr. Eduardo Duhalde, quien finalizó el período incompleto, la empresa tuvo que dedicarse a la fabricación de partes y a otros proyectos no vinculados con la aeronáutica. Finalmente, los socios de la empresa Cicaré Helicópteros S.A. dejaron de aportar a la actividad y se produjo su cierre.

En el 2003, luego de las elecciones presidenciales, asumió el cargo el Dr. Nestor Kirchner. Durante esta transición, comenzó un proceso de reactivación de la economía impulsado por el gobierno, en parte mediante el apoyo a instituciones y organismos del Estado y, además, por una fuerte apuesta a la economía de sustitución de importaciones. Además, según cuenta Wainer (2018), ocurrió una evolución favorable en términos de intercambio: una inicial contracción de las importaciones debido al desenlace de la crisis de la convertibilidad (devaluación y recesión), un aumento de las exportaciones y una reestructuración de deuda pública. Estas medidas causaron una pronta recuperación social y un fortalecimiento de la industria local.

En el 2005, se llevó a cabo una reunión entre el jefe del estado mayor del ejército, Tte. Gral. Roberto Bendini, y Augusto Cicaré. En esta, el primero manifestó el interés del ejército en el desarrollo de un helicóptero ligero experimental biplaza con turbina para uso civil, militar y de fuerzas de seguridad.

La función civil del helicóptero modelo denominado $\mathrm{CH}$-14 Aguilucho sería de entrenamiento y observación. Por su parte, el rol militar y de fuerzas de seguridad apuntaba al control de objetivos terrestres.

El programa planteado constaba de tres etapas. En la primera, se detallaban las características del prototipo surgidas de la discusión sobre los requerimientos del Ejército y las propuestas de Cicaré. La segunda etapa consistía en la fabricación y los ensayos del prototipo, a cargo de la empresa Prime Contractor. 
Finalmente, la etapa tres trataba del proceso de certificación. Puede considerarse aquí que se debía dejar a un lado el método de diseño basado en prototipos que dio origen a la trayectoria de diseño de Cicaré. Aquella metodología, similar a la planteada por Gero (1990), debía considerar ahora riesgos de incumplimiento de requerimientos que, en este caso, no serían admitidos por el cliente o por los costos generados al mismo contratista. Es decir, realizar innumerables ensayos de prueba y error erosionarían las ganancias obtenidas por la empresa de Saladillo. De manera que aquí el plantel de jóvenes profesionales y la metodología clásica de diseño (Mott, 1995) fueron la apuesta central para el desarrollo que, además, estaba dividido en etapas características de productos complejos, como las que detalla Vincenti (1990).

Si bien estaba claro que Augusto Cicaré llevaría adelante el concepto de diseño aeronáutico del helicóptero, se decidió reclutar a un grupo de jóvenes profesionales para el desarrollo del fuselaje, las condiciones aerodinámicas y la estética de la nave. El desarrollo del nuevo helicóptero y su primer prototipo comenzó en enero del 2006 y su construcción terminó hacia noviembre del mismo año bajo un régimen de trabajo que incluía horas extra y jornadas laborales la mayoría de los fines de semana. El Cicaré $\mathrm{CH}$-14 Aguilucho Experimental (figura 4) es un biplaza en tándem propulsado con turbina Rolls Royce Allison 250 desarrollado en cumplimiento de normas FAR 27. El rotor es característico de los modelos Cicaré, rotor principal y de cola, bipalas de materiales compuestos y comandos hidráulicos.

La campaña de ensayos del helicóptero transcurrió de manera normal, con Fernando Cicaré como piloto principal de pruebas. Estas se finalizaron a mediados del 2007. El 23 de noviembre del mismo año, se realizó la presentación oficial del helicóptero Cicaré $\mathrm{CH}-14$ Aguilucho en el predio del Comando de Aviación en Campo de Mayo.

En el 2008, comenzó la tercera etapa de certificación del helicóptero, a cargo del Ejército, aunque luego de la renuncia del Tte. Gral. Roberto Bendini y la asunción al cargo de jefe del estado mayor del ejército del Tte. Gral. Luis Alberto Pozzi, el proyecto entró en una pausa de varios meses. Finalmente, fue discontinuado.

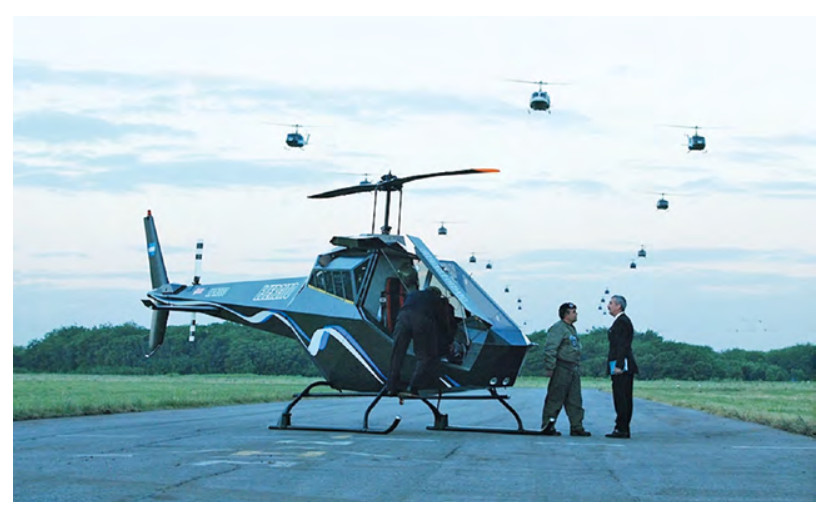

Figura 4. Prototipo modelo $\mathrm{CH}-14$ Aguilucho durante su presentación. Campo de Mayo, Buenos Aires, 2007.

Fuente: Cicaré S.A. (2016).

\section{Conclusiones}

En la fase I, entre 1956 y 1963, se puede apreciar que Cicaré consolida un proceso no convencional de desarrollo, en el que el diseño se da a través de la construcción de prototipos, la corrección y la reconstrucción. Es decir, el prototipo comienza a ser construido sin planimetría, croquizados ni análisis previos. De hecho, el banco de pruebas agiliza esa metodología y le permite no solo dedicarse a la construcción de helicópteros como sistema sino a sus partes. En el experimental, realiza ensayos sobre distintos tipos de aspas, transmisiones, comandos, materiales, instrumental, entre otros. La actividad ingenieril a partir de la construcción conforma el total de las actividades llevadas a cabo por el diseñador, como se ha descrito en Ciapuscio (1996).

Esta fase conforma los inicios en la industria aeronáutica del constructor, proveniente de la metalúrgica y las máquinas agrícolas. Esto se refleja en la adaptación de técnicas y materiales de herrería artesanal a la aeronáutica; una relación poco común, ya que las aeronaves requieren materiales optimizados en peso y resistencia. Vale la pena destacar también que esta adaptación de soluciones comunes en la industria agrícola o automotriz serían novedosas para la aeronáutica, aunque no innovadoras para el momento. Puede apreciarse aquí aquella técnica y oficio detallado anteriormente por McGinn (1991). Sin embargo, estas soluciones novedosas serían rudimentarios 
diseños conceptuales provenientes de su interpretación primitiva de las soluciones, aunque optimizados en un futuro. En definitiva, se irían formando pequeños nuevos paradigmas en soluciones aeronáuticas que la industria en general ya consideraba maduros.

Puede afirmarse que esta primera etapa se caracteriza por la necesidad de reinventar un desarrollo tecnológico debido al desconocimiento del estado del arte sobre el vuelo vertical, como destaca Mackenzie (1996). Cicaré no contaba con el conocimiento sobre aeronáutica del vuelo vertical, ni en sus aspectos explícitos (manuales, instructivos) ni en los tácitos (maniobras de vuelo, comando). De esta manera, la única alternativa era la re-generación de estos. Por su falta de formación en ciencia básica, el diseñador recurrió a la experimentación de prototipos como método central de aprendizaje, tal como lo describe Collins (2010). Además, esta era para él la única posibilidad, debido a la falta de formación académica.

En 1963, entre los desarrollos de los modelos $\mathrm{CH}-1$ y CH-2, Augusto Cicaré está en contacto con el primer helicóptero que puede observar y revisar, un Sikorsky s-51. Podría decirse que aquí se rompe esa barrera de la que habla Mackenzie y se hace contacto con un artefacto que, de alguna manera, representa un avance tecnológico tangible. Sin embargo, lo relevante aquí es tener en cuenta que el constructor no consideró que todas las soluciones mecánicas que presentaba aquel helicóptero fueran interesantes. Es cierto que muchas de ellas le parecieron complejas, pero es interesante su observación, ya que luego de aquella revisión siguió prefiriendo algunos de sus diseños artesanales sobre los industriales que ahora conocía. Definitivamente, allí el diseñador realizó una valoración de la evolución natural del primer diseño para obtener el segundo, tal como lo describe Vincenti (1990).

De más está aclarar que el diseño no se llevaba a cabo de manera estándar, el constructor no realizaba planos ni croquis, simplemente imaginaba las formas de sus objetos y los iba construyendo de esta manera. Siempre se apoyó en sus altas capacidades para la fabricación, y dejó a un lado su baja preparación en el cálculo y la representación gráfica. Esta característica fue todo un obstáculo para la formación de una empresa que apuntaba a fabricar en serie, pues Augusto nunca documentó sus desarrollos, ensayos ni resultados.

Respecto a la fase II, entre 1963 y 1990, es posible destacar que el modelo $\mathrm{CH}-6$ fue de los más importantes diseños de Cicaré por su grado de innovación. Desde el punto de vista tecnológico, fue la evolución definitiva de los modelos anteriores que llevó a la marca a ser reconocida mundialmente. Este modelo recopila de alguna manera todos los aprendizajes obtenidos en la fase I, en la que el diseñador solucionó aspectos de vuelo y de comandos. De alguna manera, además, se alcanzó aquí una maduración del aprendizaje a través de la experimentación (Collins, 2010).

Los aspectos de vuelo desarrollados durante este periodo fueron iniciales y básicos de aeronáutica: potencia de motorización, desplazamientos y aerodinámica. Los mecanismos de comando fueron los de mayor desarrollo. Definitivamente, se empezaron a caracterizar los modelos Cicaré como helicópteros de grandes flexibilidades de comando, ágiles, precisos, que incluso podían realizar acrobacias. Existió aquí una transferencia de conocimiento desde los modelos $\mathrm{CH}-1, \mathrm{CH}-2, \mathrm{CH}-3, \mathrm{CH}-4 \mathrm{Y} \mathrm{CH}-5$ con un alto componente tácito, como menciona Polanyi (1962). La relación entre las soluciones mecánicas adoptadas en los diferentes modelos y el comportamiento posterior del helicóptero muestran aquellas definiciones ostensivas que el autor menciona y que requieren de la inteligencia de quien intenta aprender para ser captadas, como la maniobrabilidad de las naves, sus reacciones, sus capacidades, entre otras. Vale la pena entonces preguntarse cómo fue posible esta transferencia. De alguna manera, esos conocimientos fueron depositados en los prototipos de aquellos modelos, ya que, como describe Polanyi (1962), muchos conocimientos no son posibles de archivar como documentación.

Puede reconocerse aquí un método de transferencia de conocimiento que contiene, además de lo explícito, un alto componente tácito, como menciona Mackenzie (1996). Cicaré intercambiaba piezas y sistemas entre sus nuevos desarrollos y los anteriores para experimentar los cambios en vuelo y corregir aspectos de manera continua. Los prototipos de todos 
sus modelos son conservados como fuente de conocimiento explícito de piezas y mecanismos. Sin embargo, para aprender aspectos tácitos, el diseñador puede probar esos mismos modelos anteriores y experimentar. Al momento de desarrollar nuevas piezas o mecanismos, estos pueden ser ensayados en los anteriores modelos de vuelo para interpretar su funcionamiento. A su vez, los prototipos le sirven al diseñador para instruir al personal de la empresa, que va adquiriendo de manera colectiva un conocimiento compartido, tal como lo detalla Collins (2010).

En esta fase, el constructor comprende la necesidad de salir de su posición de solitario inventor de tiempos libres en un taller para máquinas agrícolas y pasar a un taller pensado para el diseño y fabricación de helicópteros. Se vale además de la ayuda de otros actores como pilotos, ingenieros y técnicos especializados en aeronáutica. Es cierto que no logra afianzar este plantel y una fábrica dedicada. Pero su acercamiento a la industria, otras marcas y otros diseñadores lo hacen valorar las ventajas de la ingeniería y de las tecnologías de producción. Desde el punto de vista comercial, con el interés generado en exposiciones y convenciones aeronáuticas, Cicaré comprendió el potencial de sus diseños, y que un emprendimiento propio sería posible merced a las prestaciones de sus productos.

Finalmente, sobre esta etapa es interesante cuestionar el surgimiento de innovaciones a partir de un desarrollador aeronáutico no formado en el área, proveniente de otra especialidad, como la agroindustria. Muchas de las innovaciones fueron simplemente descubiertas por Cicaré mientras realizaba ensayos de vuelo para calificar ciertos comportamientos de los helicópteros y modificar continuamente componentes y mecanismos en búsqueda de mejoras. En otros casos, fueron pilotos expertos quienes observaron características únicas en ciertos sistemas de las aeronaves. Estas innovaciones pueden haber sido generadas, en parte, por haber reinventado una tecnología, como menciona Mackenzie (1996). En segundo lugar, apostar por la experimentación como método de aprendizaje (Collins, 2010) y de diseño permitió que los mismos prototipos, al ser continuamente modificados, sacaran a la luz nuevas funcionalidades diferentes a las inicialmente buscadas (Gero, 1990). El crédito de Cicaré y sus colaboradores es haber sabido apreciar aquellas características inusuales y valorables de los prototipos y capitalizarlas como nuevos sistemas innovadores.

Según lo detallado en la fase III, entre 1990 y 1993, y a diferencia de la anterior, Augusto Cicaré conforma un plantel de pares de confianza a partir de la concepción del modelo $\mathrm{CH}-7$. Este modelo fue la maduración definitiva y comercializable del modelo $\mathrm{CH}-6$, el cual alcanzó sus mejores prestaciones técnicas. Se aprecia aquí la experiencia más clara de evolución de diseño planteada por Vincenti (1990), más precisamente, a partir de corrección de fallas de modelos predecesores (Petroski, 1992). Se puede afirmar, desde el punto de vista de características de vuelo, que el modelo $\mathrm{CH}-7$ es el modelo final en la evolución de los desarrollos de Cicaré. A partir de este diseño se obtuvieron nuevos modelos alternativos de helicópteros de distintas prestaciones y capacidades, los cuales permitieron a la empresa ampliar su gama de productos más adelante.

Es cierto que en esta tercera fase el plantel de la empresa contaba con menor formación específica que las anteriores organizaciones. Sin embargo, en este caso se trataba de un grupo de jóvenes cercanos y de confianza, con los que Augusto Cicaré pudo, en definitiva, adaptarse a trabajar. Es aquí cuando el diseño se transforma y adopta un método comparable al descripto por Mott (1995): el proceso de desarrollo se acerca más a los pasos típicos del diseño ingenieril que plantea el autor.

Una característica notoria de esta fase son las dos experiencias de conflictos de patentes que Cicaré experimenta, en primer lugar, con Dennis Fetters y Revolution Helicopters, y, en segundo lugar, con Pierluigi Barbero y Elisport. Cicaré comprende aquí que sus soluciones son valoradas en la industria aeronáutica y tienen un gran potencial comercial y tecnológico, por lo que comienza el proceso de patentamiento de las soluciones más innovadoras. El modelo $\mathrm{CH}-7$, que fue el protagonista de este proceso y tiene las mejores características de vuelo de toda la gama de productos de la empresa, fue el de mayor volumen de ventas y el 
que evolucionó en nuevos productos que ampliaron aún más esa gama. Se hicieron desde simples adaptaciones al modelo $\mathrm{CH}-7$ que permitieron pasar de un monoplaza a un biplaza, como el CH-7B, hasta más complejas transformaciones que llevaron a desarrollar un simulador de vuelo como el svH, sin precedentes en la industria.

El simulador de vuelo SVH ha hecho, a partir de la interpretación primitiva del diseño, un producto de los más innovadores de la empresa. Es decir, a partir de la solución elemental del problema de aprendizaje de comando de vuelo desarrollada por Cicaré, que consistía en limitar la elevación de los ultralivianos con anclajes al piso, surgió un producto de aplicación internacional en la instrucción de nuevos pilotos. Esto se acerca además a la búsqueda de nuevas funcionalidades en prototipos de prueba planteada por Gero (1990). Definitivamente, se aprovechó el potencial de comercialización de un dispositivo que, en un principio, era una herramienta de trabajo para la empresa.

En la fase IV, desde 1993 hasta el 2001, se diseñan los modelos $\mathrm{CH}-8, \mathrm{CH}-11$ Y CH-12, con la particularidad de que todos ellos fueron desarrollados a partir de la estructura y los mecanismos del $\mathrm{CH}-7$. Se describe principalmente en esta fase la característica evolutiva de los diseños Cicaré, tal como lo detallaran Vincenti (1990) y Petroski (1992). Se aprecia en la trayectoria de diseño de Cicaré que cada modelo ha derivado, mediante modificaciones de ingeniería, en un nuevo modelo de vuelo mejorado o con distintas capacidades o funcionalidades. Esta fase se caracteriza principalmente por este último aspecto. Se ha ampliado la gama de productos para cubrir diferentes segmentos de la industria de los helicópteros mediante la evolución de un modelo de referencia, como el CH-7.

Finalmente, en la fase v, que describe la trayectoria entre el 2001 y el 2015, es claro que los métodos de diseño y producción artesanal se convirtieron poco a poco en una fábrica elemental. No solamente se contaba con la infraestructura y algunas tecnologías de fabricación necesarias para la producción aeronáutica, sino que además se había conformado un staff mínimo de trabajadores que comprendían los diseños y aportaban sus conocimientos. Además, estos empezaron a familiarizar al diseñador original de la empresa con la documentación estándar en la ingeniería, tal como planos, croquis, bocetos, entre otros. Aquí se fortalece la zona de negociación (Galison, 1999) y comienzan a darse análisis estructurales y verificaciones físicas de diseño, complementados con los ensayos que, en un principio, eran la única herramienta de evaluación.

Una conclusión final, y que abarca el emprendimiento desde sus comienzos hasta hoy, es que Cicaré ha logrado establecer una característica de diseño en la que conviven dos métodos de desarrollo de alta tecnología, los cuales son combinados de manera estratégica. Por un lado, la metodología de Mott (1955) es aplicada para la producción en serie de sus modelos estándares, como $\mathrm{CH}-7, \mathrm{CH}-8$, SVH 4 y CH-12. Mientras tanto, una producción basada en la metodología de prototipos de Gero (1990) genera innovaciones que apuntan a la mejora continua de productos, el surgimiento de nuevos modelos, componentes y sistemas aeronáuticos.

La interpretación primitiva (como concepto que se propone en esta investigación) de soluciones mecánicas y aeronáuticas ha sido la característica que, adaptada al proceso de transformación que se da entre las funciones a cumplir por el producto y la generación de prototipos (Gero, 1990), ha permitido a Cicaré implementar soluciones innovadoras, tanto en mecánica general como en la aeronáutica. Esto ha sido posible por la adaptación de medios a funciones preconcebidas por Augusto Cicaré; medios de la mecánica general (Ciapuscio, 1996) llevados a la industria aeronáutica que, de acuerdo a sus características específicas (Norton, 2000), han resultado sin precedentes. Vale la pena aclarar que observaciones de primera mano relacionadas con la técnica y el oficio del diseñador (McGinn, 1991) han permitido fortalecer esta característica de diseño basada en la interpretación primitiva y el estudio exhaustivo de prototipos en el proceso de desarrollo.

Quizás una de las características más notorias de la empresa, y que la diferencian de la industria típica de alta tecnología, sea la distribución física de su planta fabril. Cicaré S.A. cuenta con dos grandes edificios en su emplazamiento. En uno de ellos se encuentra 
el sector de ingeniería acoplado a la sala de producción. En el edificio lindero se erige el depósito, donde se encuentran todos los prototipos producidos por $\mathrm{Ci}$ caré, desde el $\mathrm{CH}-1$ hasta el $\mathrm{CH}-7$, pasando por motores, sistemas aeronáuticos, palas y demás componentes. Este segundo edificio, con cualidades de museo aeronáutico, permite a ingenieros y técnicos consultar soluciones adaptadas a modelos anteriores, realizar nuevos ensayos de vuelo o probar y calificar nuevos componentes y sistemas en prototipos funcionales.

Podría decirse que el conocimiento explícito se acumula en aquellos modelos almacenados, además de sus respaldos en la documentación generada en los últimos años. Además, esos prototipos pueden ser probados en vuelo para aprender de ellos aquel componente tácito que radica en la aeronáutica. Esto permite mantener todo el conocimiento generado a lo largo de la trayectoria de la empresa, asegurándose de que no sea perdido, de acuerdo a la visión alternativa de la ciencia descripta por Mackenzie (1996). Definitivamente, el estudio de prototipos es la característica principal que el método de diseño particular de Cicaré ha desarrollado y fortalecido a lo largo de los años. Se trata de una manera particular y característica de gestión de conocimiento, pues los prototipos experimentales son en sí mismos una fuente de conocimiento tecnológico, con alto componente tácito, al que puede acceder el personal de la empresa a través del estudio y ensayo de los modelos.

Mediante desarrollos innovadores que han establecido nuevos paradigmas, por ejemplo, en la formación de pilotos o en el vuelo de modelos ágiles, Cicaré ha logrado una identidad como marca dentro de la industria aeronáutica, que lo valora como uno de los líderes en helicópteros ultralivianos a nivel internacional. La empresa ha aportado un valor agregado a la industria aeronáutica a partir del diseño de helicópteros ultralivianos, un segmento poco explorado en los comienzos del inventor, lo que le demandó realizar desarrollos sin precedentes. Estas innovaciones conforman avances tecnológicos que la empresa logró capitalizar y que la industria a nivel internacional considera originales de la marca argentina.

\section{Referencias bibliográficas}

Ciapuscio, H. (1996). El conocimiento tecnológico. Revista Redes. Cicaré s.A. (2016). Productos. http://www.cicare.com.ar

Collins, H. (2010). Tacit and Explicit Knowledge. University of Chicago Press.

Galison, P. (1999). The Trading Zone: Coordinating Action and Belief. En M. Biagioli, The Science Studies Reader (pp. 782-844). Routledge.

Gero, J. (1990). Design Prototypes: A Knowledge Representation Schema for Design. Al Magazine, 11(4), 26. https:// doi.org/10.1609/aimag.v11i4.854

Layton, E. (1971). Mirror-Image Twins: The Communities of Science and Technology in 19th-Century America. John Hopkins University Press.

Mackenzie, D. (1996). Knowing Machines: Essays on Technical Change. The MIT Press.

McGinn, R. (1991). Ciencia, tecnología y sociedad. Stanford University Press.

Mott, R. (1995). Diseño de elementos de máquinas. Pearson Educación.

Nonaka, I. (1991). La empresa creadora de conocimiento. Harvard Business School Publishing Corporation.

Norton, R. (2000). Diseño de maquinaria. McGraw Hill.

Petroski, H. (1992). To engineer is human: the role of failure in successful design. St Martin's Press.

Polanyi, K. (1962). Tacit Knowing: Its Bearing on Some Problems of Philosophy. Review of Modern Physics, 34(4), 601-616. https://doi.org/10.1103/RevModPhys.34.601

Vincenti, W. (1990). What engineers know and how they know it. Johns Hopkins University Press.

Wainer, A. (2018). Economía y política en la Argentina kirchnerista (2003-2015). Revista Mexicana de Sociología, 80(2), 323-351. http://dx.doi.org/10.22201/iis.01882503 p.2018.2.57720 